\title{
Low levels of methylmercury induce DNA damage in rats: protective effects of selenium
}

\author{
Denise Grotto • Gustavo R. M. Barcelos • Juliana Valentini • \\ Lusânia M. G. Antunes · José Pedro F. Angeli • \\ Solange C. Garcia $\cdot$ Fernando Barbosa Jr
}

Published online: 26 November 2008

(C) Springer-Verlag 2008

\section{Erratum to: Arch Toxicol}

\section{DOI 10.1007/s00204-008-0353-3}

Unfortunately, in this article there is a mistake in the title of one of the references.

The paper referred as

Franco JL, Braga HC, Stringari J, Missau FC, Posser T, Mendes BG, Leal RB, Santos AR, Dafre AL, Pizzolatti MG, Farina M (2007) Comparative study of activities in reactive oxygen species production/defense system in mitochondria of rat brain and liver, and their susceptibility to methylmercury toxicity. Chem Res Toxicol 20:1919-1926

should read

Franco JL, Braga HC, Stringari J, Missau FC, Posser T, Mendes BG, Leal RB, Santos AR, Dafre AL, Pizzolatti MG, Farina M (2007) Mercurial-induced hydrogen peroxide generation in mouse brain mitochondria: protective effects of quercetin. Chem Res Toxicol 20:1919-1926.

The online version of the original article can be found under doi: 10.1007/s00204-008-0353-3.

D. Grotto · G. R. M. Barcelos · J. Valentini - L. M. G. Antunes

F. Barbosa $\operatorname{Jr}(\square)$

Departamento de Análises Clínicas,

Toxicológicas e Bromatológicas, Faculdade de Ciências

Farmacêuticas de Ribeirão Preto, Universidade de São Paulo, Avenida do Café s/n, Campus Universitário da USP,

14040-903 Ribeirão Preto, São Paulo, Brazil

e-mail: fbarbosa@fcfrp.usp.br

J. Valentini · S. C. Garcia

Departamento de Análises Clínicas e Toxicológicas,

Universidade Federal de Santa Maria, Av. Roraima 1000,

Cidade Universitária, CEP 97105-900 Santa Maria,

Rio Grande do Sul, Brazil

J. P. F. Angeli

Departamento de Bioquímica, Instituto de Química,

Universidade de São Paulo, Av. Professor Lineu Prestes 748,

CEP 05508-900 São Paulo, Brazil 